\title{
Can Hubble tension be eased by invoking a finite range for gravity?
}

\author{
Louise Rebecca \\ Christ University, Bangalore, 560 095, India \\ email: louiserheanna.rebecca@res.christuniversity.in
}

\section{Kenath Arun ${ }^{1}$}

Christ University, Bangalore, 560 095, India

e-mail: kenath.arun@christuniversity.in

\author{
C Sivaram \\ Indian Institute of Astrophysics, Bangalore, 560 034, India \\ e-mail: sivaram@iiap.res.in
}

\begin{abstract}
The estimation of the Hubble constant in the past few decades has increasingly become more accurate with the advance of new techniques. But its value seems to depend on the epoch at which the measurements are made. The Planck estimate of the Hubble constant from the observations of the CMBR in the early Universe is about $67 \mathrm{kms}^{-1} \mathrm{Mpc}^{-1}$, whereas that obtained from the distance indicators at the current epoch is $\sim 73-74 \mathrm{kms}^{-1} \mathrm{Mpc}^{-1}$. This discrepancy between the two groups of measurement is termed as the Hubble tension which has gained much attention in the past few decades with growing significance as measurements from both, the early and the late Universe, studies continue to produce results with increasing precision. Here we propose a modification to gravity by considering a finite range gravitational field as an alternate explanation for this discrepancy in the value of the Hubble constant.
\end{abstract}

Keywords: Hubble constant; Hubble tension; early Universe; modified gravity; finite gravity

\footnotetext{
${ }^{1}$ Corresponding author
} 


\section{Introduction}

One method of estimation of the Hubble constant is by observation of anisotropies and other features of the cosmic microwave background radiation, which provides a snapshot of the early Universe. Another process involves estimation of the Hubble constant 'locally' by determining the distances to nearby galaxies through their distance-redshift relation. These methods involve calibration of luminosities of standard candles such as Cepheid variables, type Ia supernovae, tip of red giant branch (TRGB), etc. The late Universe measurements give a value of Hubble constant that is significantly different from that measured from the CMBR [17]. It is evident with these observational results that this discrepancy in measured value of Hubble constant is not due to observational errors. Hence, we need a theoretical framework to explain this apparent inconsistency.

\section{Hubble Constant Measurements}

The very first measurement of the Hubble constant $\left(H_{0}\right)$ - by Hubble himself - used Cepheid variables as standard candles yielding a value of about $500 \mathrm{kms}^{-1} \mathrm{Mpc}^{-1}$ [8]. This value is found to be more than five times the value obtained by subsequent measurements over the past several decades. The Hubble Space Telescope's key project established the most precise determination of $H_{0}$ with secondary distance measurements over a range of $60-$ $400 \mathrm{Mpc}$ with Cephids as standard candles. The project yielded a $H_{0}$ value of $72 \pm$ $8 k m s^{-1} M p c^{-1}$

There has been further progress in reducing known systematic errors in measurements with the increase in the samples of Type Ia supernovae [9-12]. The Wide Field Camera 3 on the Hubble Space Telescope was used to reduce the uncertainty from 3.3\% to $2.4 \%$. Most of this improvement comes from recent near-infrared observations of Cepheid variables in eleven galaxies hosting recent type Ia supernovae. This observation more than doubled the sample of 
reliable type Ia to a total of 19. From this, the best estimate of Hubble constant is found to be $73.24 \pm 1.74 \mathrm{kms}^{-1} \mathrm{Mpc}^{-1}$ [9]. New measurements [13] involving the late Universe reinforced the Hubble value $H_{0}$ obtained from the results of Supernova for the Equation of State (SH0ES) project $[7,14]$. The late Universe measurements have also improved distance estimates with the usage of detached eclipsing binaries, water masers, etc., all of which produce values of $H_{0}$ in the range of $72-73 \mathrm{kms}^{-1} \mathrm{Mpc}^{-1}[15]$.

Another independent measurement used alternate distance ladders such the Tip of the Red Giant Branch (TRGB) rather than Type Ia supernova producing a $H_{0}$ value of $69.8 \pm$ $0.8 \mathrm{kms}^{-1} \mathrm{Mpc}^{-1}[16]$, midway between the values from Planck and SH0ES. The most recent calibration of Type Ia supernova based on the surface brightness fluctuations (SBF) method consisting of 24 supernovae hosted in galaxies that have SBF distance measurements estimated a value of $70.50 \pm 3.38 \mathrm{kms}^{-1} \mathrm{Mpc}^{-1}$ [17]. This is in good agreement with that obtained from the TRGB calibration.

The standard-candle method is not the only method that can be used to obtain $H_{0}$. A series of high-resolution images of the Cosmic Microwave Background Radiation was captured by the Wilkinson Microwave Anisotropy Probe (WMAP) [18] as an independent measure of the rate of expansion of the 'early Universe'. While initial results seemed to be almost in agreement with that obtained from the 'late Universe', this agreement is seen to disappear with increased refinement in the method. The nine-year data release from WMAP produced a $H_{0}$ of $69.32 \pm 0.80 \mathrm{kms}^{-1} \mathrm{Mpc}^{-1}[19]$. This was followed by the Planck Mission [20-22] that acquired the highest resolution maps to date in microwaves. Analysis of fluctuations in temperature and polarization maps lead to an excellent agreement with the standard model of cosmology. Fitting the angular fluctuations in the Planck data to the $\Lambda \mathrm{CDM}$ model results in a $H_{0}$ value of $67.8 \pm 0.9 \mathrm{kms}^{-1} M p c^{-1}$. The Baryonic Accosting Oscillations method (BAO) in collaboration with Baryon Oscillations Spectroscopy Survey (BOSS) produced a value of a $H_{0}$ 
of $67.3 \pm 0.9 \mathrm{kms}^{-1} \mathrm{Mpc}^{-1}$ independent of CMB anisotropies [23-25]. Recent measurements independent of both, the distance ladder and CMB, such as the $\gamma$-ray attenuation measurements from Fermi-LAT [26] produced a value of $66.6 \pm 1.6 \mathrm{kms}^{-1} \mathrm{Mpc}^{-1}$.

As Hubble constant measures the rate of expansion of the Universe, the above values suggest that the Universe is expanding faster at present than at the time of the CMBR epoch. Sources of systematic error could only increase the value of $H_{0}$ from Planck by not more than $1 \mathrm{kms}^{-1} \mathrm{Mpc}^{-1}$ under the $\Lambda \mathrm{CDM}$ model. Thus, Hubble tension, even if reduced would probably continue to exist.

\section{Finite Range Gravity (FRG)}

Although general relativity is well established through several observations, certain cosmological observations such as the accelerated expansion, missing matter (dark matter) and the recent tension in the Hubble constant measurements probably necessitates a need for slight modifications to the theory. One such modification can be done by considering gravity to be of a finite range. There have been suggestions of a finite range gravity earlier, one such suggestion involved specific mass terms (masses of spin- 2 and spin- 0 gravitons) in addition to the field-theoretical analogue of the usual Hilbert-Einstein Lagrangian that allows the cosmological scale factor to exhibit an accelerated expansion [27]. There have also been suggestions of a multidimensional higher derivative theories of gravity that is marked by a nonpolynomial form factor which averts extra degrees of freedom. In odd dimensions it is seen that gravity turns out to be 'finite' [28]. Finite gravity was also suggested in another context [29, 30]. For a review see for e.g. [31].

The tension in the Hubble constant between different cosmological epochs - if not due to errors in measurement - may be an actual indication of the present expansion rate of the Universe. Here we consider gravity to be of a finite range that could possibly account for an 
increased expansion rate in the present epoch. With this model, the gravitational potential is modified as

$V=-\frac{G M}{r}-\frac{G M}{r} e^{\frac{-r_{0}}{r}}$

where $r_{0}$ is a constant, indicating the range. If gravity is of finite range, it will weaken following equation (1) as $r$ becomes large.

The Friedmann equation, now gets modified to

$H^{2}=\frac{\dot{R}^{2}}{R^{2}}=\frac{8 \pi G \rho}{3}+\frac{8 \pi G \rho}{3} e^{\frac{-r_{0}}{r}}+\frac{\Lambda C^{2}}{3}$

The second term in the expression changes the expansion rate by $e^{\frac{-r_{0}}{r}}$ as the Universe undergoes expansion from $r_{0}$ to $r$. The second term becomes important at $r \geq r_{0}$. For the present epoch, with $r=10^{28} \mathrm{~cm}$, for a decrease in the value of the second term by $10 \%$ (i.e., $e^{\frac{-r_{0}}{r}} \sim 0.1$ ), the value of $r_{0}$ has to be $\approx 5 \times 10^{27} \mathrm{~cm}$. This range for the gravitational field corresponds to a graviton mass which may be related to the cosmological constant through

$m_{\text {grav }}=\frac{h \sqrt{\Lambda}}{c} \approx 2 \times 10^{-65} \mathrm{~g}$

This thereby implies that as $r \rightarrow r_{0}$ gravity can weaken and $H^{2}$ can become $10 \%$ larger indicating an increase in the rate of expansion. This could probably account for higher value of Hubble constant seen at the present epoch.

Note: Indeed a potential of the form given by equation (1) arises in the Newtonian limit of the modified actions of the form $R+R^{2}$, i.e., a modified Poisson equation, $\nabla^{4} \phi+\nabla^{2} \phi=$ constant, with a solution of the form, $\phi \propto \frac{1}{r}\left(1-e^{-k r}\right)$. [29, 31, 32]

\section{Conclusion}

In view of the fact that the observed inconsistency in Hubble constant is not due to observational errors, we need to look for alternate models to reduce this tension. In an earlier work we invoked a modification of the gravitational field on large scales (low accelerations) 
that could provide a possible solution for this discrepancy and perhaps help in relaxing the Hubble tension [33]. In this work we extend the idea through a modification to gravity by invoking a finite range gravitational field to explain the discrepancy. Finite gravity has been introduced in other contexts earlier. Here the introduction of an exponential term to the gravitational potential modifies the Friedmann equation and this new term changes the expansion rate as the Universe expands beyond a critical size. We also suggest that the gravitational field's range corresponds to a graviton mass and this may be related to the cosmological constant. With this modified gravity we can account for the $\sim 10 \%$ change in the Hubble constant measured 'locally' versus that measured at early epochs by Planck satellite.

\section{References}

[1] E. J. Baxter and B. D. Sherwin, Mon. Not. R. Astron. Soc. 501 (2021) 1823.

[2] S. Mukherjee et al., arXiv:2009.14199 [astro-ph.CO] (2020).

[3] J. Soltis, S. Casertano and A. G. Riess, arXiv:2012.09196 [astro-ph.GA] (2020).

[4] A. G. Riess et al., arXiv:2012.08534 [astro-ph.CO] (2020).

[5] K. Dutta et al., Phys. Rev. D 100 (2019) 103501.

[6] W. Freedman et al., Astrophys. J. 882 (2019) 34.

[7] D. Alberto et al., Astrophys. J. 885 (2019) 137.

[8] E. Hubble, PNAS 15 (1929) 168.

[9] A. G. Riess et al., Astrophys. J. 876 (2019) 85.

[10] G. Pietrzynski et al., Nature 567 (2019) 200.

[11] A. G. Riess et al., Astrophys. J. 861 (2018) 126.

[12] A. G. Riess et al., Astrophys. J. 855 (2018) 136.

[13] G. C-F. Chen et al., arXiv:1907.02533 [astro-ph.CO] (2019).

[14] S. Birrer et al., Mon. Not. R. Astron. Soc. 484 (2018) 4726. 
[15] M. J. Reid et al., arXiv:1908.05625 [astro-ph.GA] (2019).

[16] W. L. Freedman et al., Astrophys. J. 882 (2019) 34.

[17] N. Khetan et al., Astron. Astrophy. 647 (2021) A72.

[18] N. Jarosik et al., Astrophys. J. Suppl. Ser. 192 (2011) 14.

[19] C. L. Bennett et al. Astrophys. J. Suppl. Ser. 208 (2013) 20.

[20] P. A. R. Ade et al., Astron. Astrophys. 594 (2016) A13.

[21] N. Aghanim et al., arXiv:1807.06209v2 [astro-ph.CO] (2018).

[22] N. Aghanim et al., Astron. Astrophys. 641 (2020) A6.

[23] J. Ryan, Y. Chen and B. Ratra, Mon. Not. R. Astron. Soc. 488 (2019) 3844.

[24] E. Aubourget et al., Phys. Rev. D 92 (2015) 123516.

[25] J. N. Greib et al., Mon. Not. R. Astron. Soc. 467 (2017) 2085.

[26] A. Domínguez et al., arXiv:1903.12097 [astro-ph.CO] (2019).

[27] S. V. Babak and L. P. Grishchuk, Int. J. Mod. Phys. D 12 (2003) 1905.

[28] L. Modesto, arXiv:1305.6741 [hep-th] (2013).

[29] C. Sivaram and K. P. Sinha, Prog. Theor. Phys. 55 (1976) 4.

[30] E. A. Lord, K. P. Sinha and C. Sivaram, Prog. Theor. Phys. 52 (1974) 161.

[31] C. Sivaram and K. P. Sinha, Phys. Rep. 51 (1979) 3.

[32] K. S. Stelle, Gen. Relativ. Gravitation 9 (1978) 353.

[33] C. Sivaram, K. Arun and L. Rebecca, Indian J. Phys. DOI: 10.1007/s12648-021-02080$7(2021)$ 